\title{
Effect of Jianpibufei plaster on chronic obstructive pulmonary disease in mice
}

\author{
Ying HUANG ${ }^{1,2}$, Bin WAN², Zhenyan $\mathrm{HUANG}^{2}$, Mian $\mathrm{LIN}^{2 *}$ (D)
}

\begin{abstract}
To explore effect of Jianpibufei plaster (JP) on chronic obstructive pulmonary disease (COPD) in mice model. The COPD mice model was established by passive smoking and intratracheal instillation of lipopolysaccharide (LPS). After treatment with JP, the body weight of mice were increased in comparison with those in the COPD group. The levels of RI, Cchord and FRC of mice in the JP group were significantly decreased when compared to those in the COPD group $(p<0.05$, respectively). In addition, JP notably attenuated the pulmonary pathological lesions and bronchiectasis. Furthermore, the levels of KC, TNF- $\alpha$ and IL-6 in BALF were markedly decreased in the JP group when compared to those in the COPD group ( $p<0.05$, respectively). Jianpibufei plaster has a protective effect on COPD mice through improving the lung function and reducing the number of inflammatory cells and the levels of various inflammatory factors.
\end{abstract}

Keywords: Jianpibufei plaste; chronic obstructive pulmonary disease; lung function; inflammation.

Practical Application: Protection of chronic obstructive pulmonary disease by Jianpibufei plaster.

\section{Background}

Chronic obstructive pulmonary disease (COPD) is a kind of chronic bronchitis and / or emphysema with the characteristics of airflow obstruction, which can be further developed into a common chronic disease of pulmonary heart disease and respiratory failure (Hogea et al., 2020). It is related to the abnormal inflammatory reaction of harmful gases and particles (Ko \& Sin, 2020). In recent years, with the aggravation of environmental pollution, COPD is threatening people's health more and more seriously, which has become a frequent and common disease. The morbidity of patients over 40 years old in the world has reached $9-10 \%$ (Dean, 2017). The pathogenesis of COPD is related to chronic inflammation of airways and lung tissue caused by harmful gases such as smoke or harmful particles (Postma et al., 2015). Many patients with COPD frequently experience acute exacerbations frequently every year, leading to a sharp decline in lung function, physical fitness and mobility, and an increase in the financial burden of hospitalization, which seriously affects work and life (Selzler et al., 2020).

The core of COPD management is to adhere to long-term standardized stable treatment, the most important of which is the long-term maintenance of the stable period of inhaled drugs. However, it is difficult to change the decline trend of pulmonary function in patients with COPD after treatment with simple chemical therapy. The new inhalers are not included in social insurance in China, so that the burden of patients is heavy because of the poor long-term effect and the high treatment cost. Some studies have shown that traditional Chinese medicine cannot only slow down the decline of pulmonary function, but also significantly reduce the production of alveolar inflammatory factors, inhibit airway remodeling, and regulate the immune function of patients with COPD by through applying medicine according to indications (Zhong, 2011; Meng et al., 2015; Vidal et al., 2020). Plaster formula is a traditional Chinese medicine, with a history of thousands of years. It combines the treatment and prevention of diseases, and has a certain clinical effect in chronic diseases. Jianpibufei plaster (JP) is made from a variety of Chinese medicinal herbs. Studies have shown that JP pill has protective effect on the skeletal muscle of COPD rats (Li et al., 2018; Mao et al., 2018). However, the specific mechanism of JP on COPD is not clear. The purpose of this study was to explore the effect of JP on COPD mice induced by passive smoking and intratracheal instillation of lipopolysaccharide (LPS).

\section{Materials and methods}

\subsection{Chemicals}

Lipopolysaccharide (LPS) was purchased from Sigma Chemical Co. (St. Louis, MO, USA). Jianpibufei plaster formula, including Radix Astragali 300 g, Codonopsis pilosula 250 g, Poria cocos 200 g, bighead atractylodes rhizome 200 g, Lily 200 g, Atractylodes $150 \mathrm{~g}$, roasted licorice $100 \mathrm{~g}$, Rhizoma Pinellinae Praeparata 100 g, dried ginger 100 g, Fritillaria thunbergii $150 \mathrm{~g}$, northern almond $100 \mathrm{~g}$, Platycodon grandiflorum $100 \mathrm{~g}$, Rhizoma Polygonatum 150 g, Magnolia obavata 150 g, cooked land $150 \mathrm{~g}$, Cornus officinalis $150 \mathrm{~g}$, lepidium $150 \mathrm{~g}$, Rehmannia glutinosa Libosch $150 \mathrm{~g}$, angelica $100 \mathrm{~g}$, Rhizoma Dioscoreae $200 \mathrm{~g}$, rhizoma anemarrhenae $150 \mathrm{~g}$, herba lycopi $150 \mathrm{~g}$, Sichuan Safflower $50 \mathrm{~g}$, banksia rose $100 \mathrm{~g}$, Amomum $50 \mathrm{~g}$, tangerine 
peel $50 \mathrm{~g}$, tortoise shell glue $150 \mathrm{~g}$, donkey hide gelatin $150 \mathrm{~g}$, and crystal sugar $200 \mathrm{~g}$ was provided by the Chinese pharmacy of Zhongshan Hospital of traditional Chinese Medicine. Hongmei cigarettes were purchased from Guangdong Zhongyan Industry Co., Ltd (Guangzhou, China). The ELISA kits of TNF-a, IL-6 and $\mathrm{KC}$ were purchased from Shanghai Guangrui Biotechnology Co., Ltd (Shanghai, China).

\subsection{Preparation of Jianpibufei plaster}

Calculate the dosage according to the number of animals and administration time. Take all the medicines in the plaster formula and soak them in an appropriate amount of water for $24 \mathrm{~h}$, then cook for juice, and filter it with absorbent cotton and gauze for 3 times. After the filtrate was mixed, it was heated with asbestos mesh and concentrated to about $2000 \mathrm{~mL}$. Add $200 \mathrm{~g}$ crystal sugar to refine the plaster. Finally, $1.26 \mathrm{~g}$ of crude drug was contained in every $1 \mathrm{~mL}$ of plaster, which was sterilized and cooled, and stored in a $4{ }^{\circ} \mathrm{C}$ refrigerator.

\subsection{Animals and protocols}

This study was approved by the institutional ethical committee of Guangzhou Institute of respiratory diseases (GIRD). A total of forty SPF eight-week-old C57BL/6J mice, weight 20-22 g, were purchased from Beijing huafukang Biotechnology Co., Ltd (Beijing, China) [SCXK[Jing]2014-0004]. Mice were raised in SPF animal laboratory of experimental animal center of GIRD (SYXK[Yue]2011-0074) with (22 \pm 2$){ }^{\circ} \mathrm{C},(50-60) \%$ relative humidity and a $12 \mathrm{~h}$ light/dark cycle. All mice were allowed free access to a standard pellet diet.

Forty C57BL/6J mice were randomly divided into five groups, namely the control group, the COPD model group, the JP with high-dose, middle-dose and the low-dose groups. The COPD mice model was established by passive smoking and intratracheal instillation of lipopolysaccharide (LPS) as previous described with some modification (Song \& Kelsen, 2013; Zhang et al., 2012). The mice, except those in the control group, were placed in the fumigating box for artificial passive smoking. The duration of smoking was 4 times a day, 2 times in the morning and afternoon respectively with 10 cigarettes/h and $1 \mathrm{~h}$ each time. The interval of smoking was $15 \mathrm{~min}$, and the interval of morning and afternoon was 3-4 h. The whole smoking lasts for 12 weeks with 6 days in a week. In addition, LPS was injected into the trachea of mice on the 1st and 14 th day, the dosage was $7.5 \mu \mathrm{g} / 50 \mu \mathrm{L} /$ animal. After successfully establishing the COPD model, the mice in the JP groups were given JP plaster solution with the concentration of $20.8,10.4$ and $5.2 \mathrm{~mL} / \mathrm{kg} / \mathrm{d}$, respectively. The dose of gavage was $0.5 \mathrm{~mL} /$ time/day and lasted for 4 cycles ( 4 weeks). At the same time, mice in the control and model groups were given administration of distilled water in the same volume. After each cycle, the body weight of mice should be measured.

\subsection{Evaluations of pulmonary function}

Four weeks later, the lung function of mice in each group was measured, including inspiratory resistance (RI), static lung compliance (Cchord) and functional residual volume (FRC). Mice were anesthetized by intraperitoneal injection of $1 \%$
Pentobarbital Sodium (100 mg/kg for the control group and $70 \mathrm{mg} / \mathrm{kg}$ for other groups). The trachea was cut at the thyroid gland of the neck. After intubation, the trachea was put into the body tracing box, and the FinePointe ${ }^{\mathrm{TM}}$ PET type small animal lung function instrument (Buxco company, USA) was connected for mechanical ventilation. Set the tidal volume of $10 \mathrm{~mL} / \mathrm{kg}$, inspiratory pressure of $8-12 \mathrm{cmH}_{2} \mathrm{O}$, breath for 120 times/min, and inspiratory/expiratory ratio of 10:15. After the figure was stable, we started to measure the pulmonary ventilation function. After the experiment, mice were killed by cervical dislocation.

\subsection{Determination of the total number of inflammatory cells and the levels of TNF-a, IL-6 and KC in Broncho Alveolar Lavage Fluid (BALF)}

After anesthesia, the airway was exposed and ligated by endotracheal intubation with indwelling needle. Open the chest and expose both lungs, then wash the Broncho alveolar with PBS buffer containing EDTA, and recover the BALF. The obtained BALF solution was centrifuged at $1500 \mathrm{r} / \mathrm{min}$ for $10 \mathrm{~min}$, and the supernatant was collected and stored at $-80{ }^{\circ} \mathrm{C}$. The levels of TNF-a, IL- 6 and $\mathrm{KC}$ in the supernatant were measured with ELISA kits. Add $200 \mu \mathrm{L}$ of red blood cell (RBC) lysate to the remaining precipitate, mix well, react at room temperature for 3-5 $\mathrm{min}$, and centrifuge at $1500 \mathrm{r} / \mathrm{min}$ for $10 \mathrm{~min}$. Add $1 \mathrm{~mL}$ PBS buffer to the precipitate, mix well, and take $10 \mu \mathrm{L}$ to the cell counting board to count the cells (Li et al., 2015).

\subsection{Histopathological analysis}

Hematoxylin and eosin staining (HE) was performed as previously reported (Wu et al., 2016). After taking the BLAF, lung tissues were collected from mice and fixed with $10 \%$ neutral formaldehyde for HE staining. The pathological changes of trachea, blood vessels and alveoli were observed under an optical microscope (OLYMPUS BX41, OLYMPUS, Tokyo, Japan) and photographed at $100 \times$ magnification.

\subsection{Statistical analysis}

All experiments were repeated three times with the same sample. Statistical analysis was made by software SPSS17.0 (International Business Machines, corp., Armonk, NY, USA). Significant differences between groups were assessed by Independent-sample t-test. All data were expressed as means \pm standard deviation (SD). Differences were considered to be statistically significant when $p<0.05$.

\section{Results}

\subsection{General behaviors observation}

Mice in the control group and JP high-dose group had good mental state, smooth, clean and glossy hair. Compared with the control group, the mice in the model group had poor mental state, slow movement, shortness of breath, lack of luster of hair with local shedding and reduced food intake. The body weight in the control group was increased when the body weight of mice in the JP high-dose group was slowly increased during 
experiment (Table 1). However, the body weight in other three group was decreased in various degrees (Table 1).

\subsection{JP improved lung function in COPD mice}

We observed the airway resistance (RI), pulmonary static compliance (Cchord), and functional residual capacity (FRC) of mice in each group to evaluate their lung function. Compared with the normal group, the levels of RI, Cchord and FRC in the COPD model group were significantly higher $(p<0.01)$ (Figure 1). However, the levels of RI, Cchord, and FRC in the JP groups were notably decreased than those in the COPD model group with a dose-dependent manner $(p<0.01)$ (Figure 1$)$.

\subsection{JP improved histopathology of lung tissue in COPD mice}

HE staining was used to detect the pathological changes of lung tissue in each group. Compared with the control group, there were

Table 1. The changes of body weight in each group before and after treatment.

\begin{tabular}{lcccccc}
\hline \multicolumn{1}{c}{ Groups } & Dose $(\mathrm{g} / \mathrm{kg})$ & 0 week & 1 week & 2 week & 3 week & 4 week \\
\hline Control & - & $22.4 \pm 1.3$ & $24.8 \pm 0.9$ & $26.4 \pm 1.1$ & $27.8 \pm 1.4$ & $29.6 \pm 1.5$ \\
COPD & - & $22.9 \pm 1.5$ & $21.7 \pm 1.1$ & $20.8 \pm 0.8$ & $20.1 \pm 0.6$ & $19.6 \pm 1.2$ \\
JP high-dose & 20.8 & $22.1 \pm 1.0$ & $22.3 \pm 1.3$ & $22.7 \pm 0.7$ & $23.1 \pm 0.8$ & $24.6 \pm 1.7$ \\
JP middle-dose & 10.4 & $21.7 \pm 1.3$ & $21.8 \pm 1.5$ & $21.4 \pm 1.8$ & $21.7 \pm 0.9$ & $21.5 \pm 1.1$ \\
JP low-dose & 5.2 & $22.6 \pm 0.7$ & $22.3 \pm 1.1$ & $21.9 \pm 0.8$ & $21.6 \pm 1.0$ & $21.1 \pm 1.6$ \\
\hline
\end{tabular}
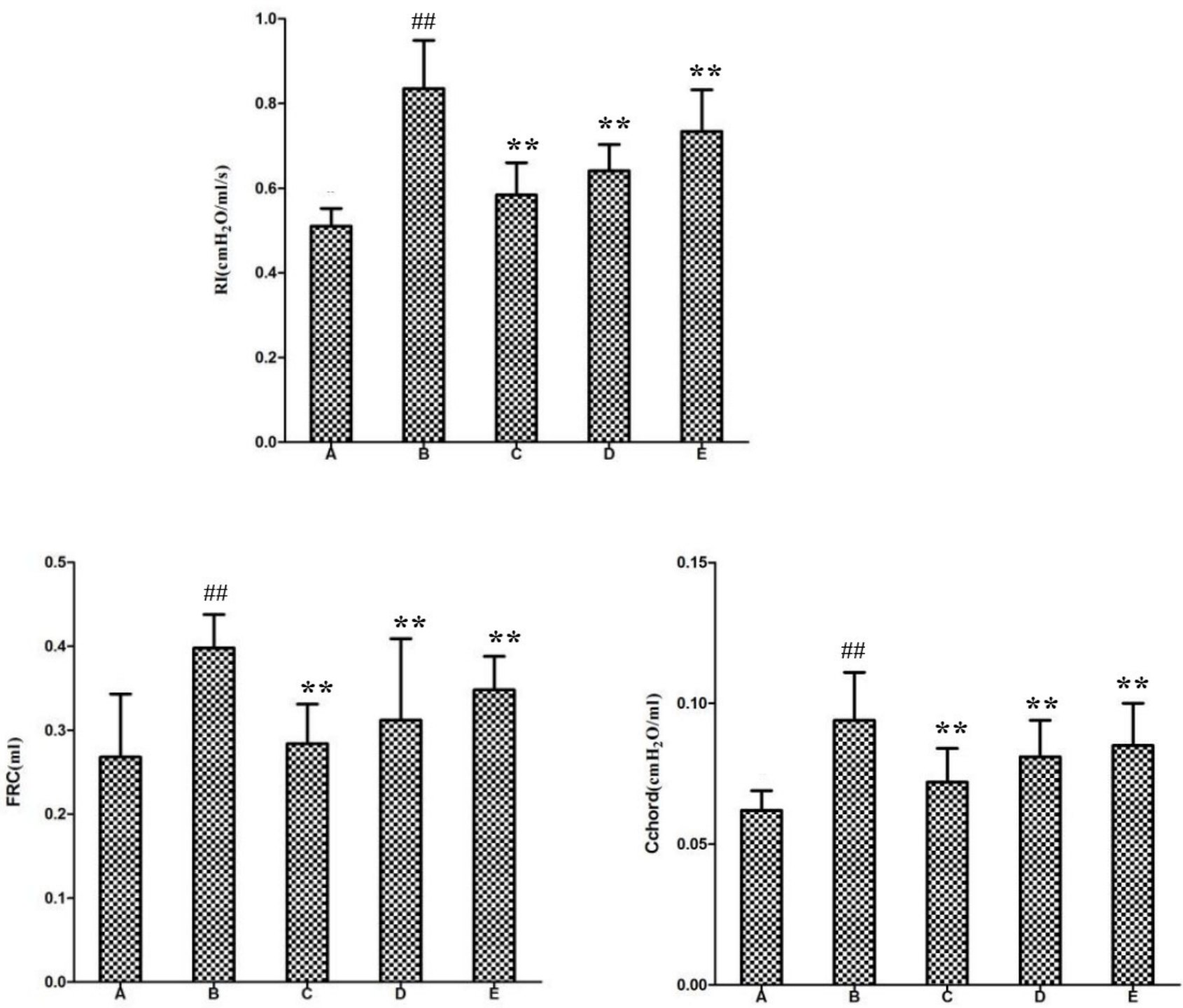

Figure 1. Effect of JP on lung function of COPD mice. (A) Control group; (B) COPD model group; (C) JP high-dose group; (D) JP medium-dose group; (E) JP low-dose group. ${ }^{* *} p<0.01$ vs. COPD model group; ${ }^{* *} p<0.01$ vs. control group. 
inflammatory cell infiltration, edema, mucus gland enlargement and mucus secretion, bronchioles expansion and destruction, alveolar cavity enlargement and formation of pulmonary bullae in the lung tissue in the COPD model group (Figure 2). In addition, the vascular wall was thickened, smooth muscle was proliferated, lung injury was more serious, and the number of pulmonary bullae was increased in COPD model mice (Figure 2). The treatment of JP obviously improved pulmonary pathological changes and inflammatory lesions in COPD mice (Figure 2).

\subsection{JP reduced the total number of inflammatory cells and the levels of inflammatory factors in BALF of COPD model mice}

We first measured the total number of inflammatory cells in BALF in each group. The results showed that the number of inflammatory cells in COPD model mice were markedly increased when compared to those in the control group, which were significantly decreased in the JP groups $(p<0.01)$ (Table 2). Furthermore, we also detected the levels of IL-6, KC and TNF- $\alpha$ in BALF. The results suggested that the levels of IL-6, KC and TNF- $\alpha$ in the COPD model group were significantly higher in comparison with those in the control group, which were notably lower after treatment with JP in a dose-dependent manner $(p<0.01)$ (Table 2).

\section{Discussion}

COPD belongs to the category of "cough" and "lung distention" in the name of traditional Chinese medicine disease (Zhang et al., 2012). Dialectically, COPD belongs to the syndrome of "deficiency of essence" and "excess of essence". In the early stage, COPD mainly refers to the deficiency of lung qi, then involves the spleen and kidney, resulting in the deficiency of the

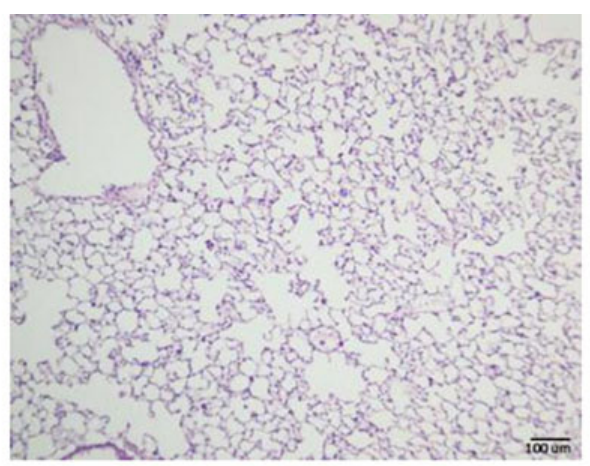

A

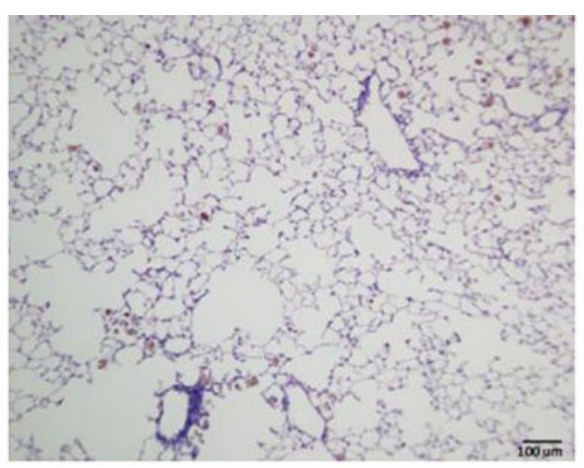

B

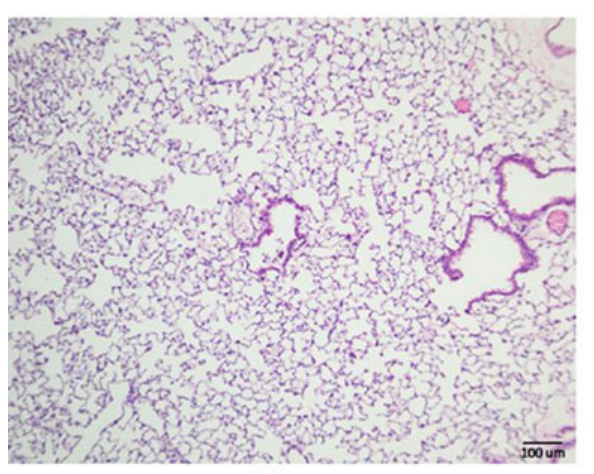

C

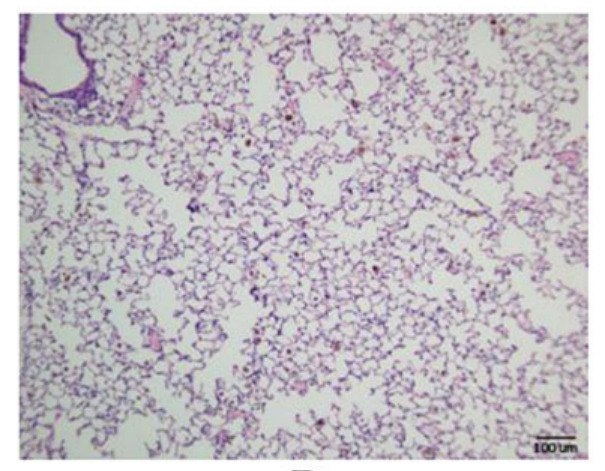

D

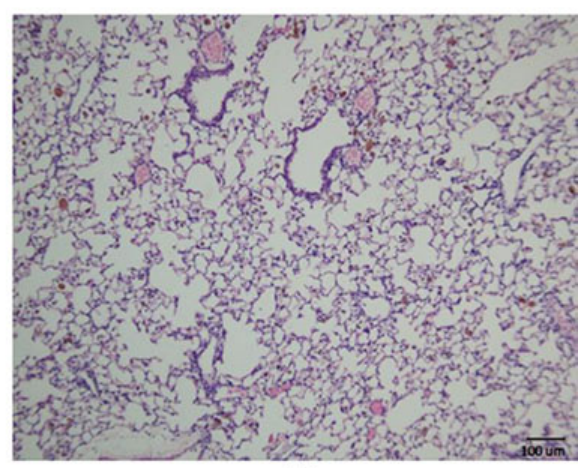

$\mathbf{E}$

Figure 2. H\&E staining of lung sections. (A) Control group; (B) COPD model group; (C) JP high-dose group; (D) JP medium-dose group; (E) JP low-dose group.

Table 2. Effect of JP on the inflammation in the lung of COPD model mice.

\begin{tabular}{lccccc}
\hline \multicolumn{1}{c}{ Groups } & Dose $(\mathrm{g} / \mathrm{kg})$ & $\begin{array}{c}\text { total cells counted } \\
\left(10^{4} \text { cells/mL }\right)\end{array}$ & IL-6 (ng/L) & KC (ng/L) & TNF- $\alpha(\mathrm{ng} / \mathrm{L})$ \\
\hline Control & - & $16 \pm 1.8$ & $7.53 \pm 0.89$ & $45.47 \pm 1.24$ & $32.8 \pm 3.4$ \\
COPD & - & $136 \pm 19.7^{* *}$ & $19.59 \pm 2.47^{* *}$ & $157.46 \pm 8.75^{* *}$ & $85.9 \pm 9.6^{* *}$ \\
JP high-dose & 20.8 & $37 \pm 1.5^{\# \#}$ & $10.16 \pm 1.13^{\# \#}$ & $66.39 \pm 6.77^{\# \#}$ & $56.1 \pm 6.8^{\# \#}$ \\
JP middle-dose & 10.4 & $58 \pm 3.8^{\# \#}$ & $12.58 \pm 1.07^{\# \#}$ & $76.47 \pm 9.48^{\# \#}$ & $67.7 \pm 7.9^{\# \#}$ \\
JP low-dose & 5.2 & $89 \pm 6.4^{\# \#}$ & $16.13 \pm 2.01^{\# \#}$ & $96.19 \pm 7.18^{\# \#}$ & $75.6 \pm 8.2^{\# \#}$ \\
\hline
\end{tabular}

${ }^{* *} p<0.01$ vs. COPD model group; ${ }^{* *} p<0.01$ vs. control group. 
three organs of lung, spleen and kidney. The standard condition is that the sputum, heat, and blood stasis are mixed and caused by disease, and the disease is difficult to heal, and the stable phase is dominated by lung, spleen and kidney failure. According to traditional Chinese medicine, the pathogenesis of COPD in stable stage is characterized by the disappearance of external pathogenic factors. This disease is located in the lung first, then affects the spleen and kidney, and the heart later.

The plaster formula can be said to have unique advantages in the treatment of COPD in the stable stage (Li et al., 2014). Based on the characteristics of deficiency of both lung and spleen in the stable period of COPD, the therapeutic effect of JP plaster on COPD models mice was studied.

Chronic airway inflammation is one of the important pathological features of COPD. COPD is always accompanied by inflammatory response, so the core of the treatment of COPD is to control inflammation. Even in the stable stage of COPD, there is airway inflammation. When the defense function of the upper respiratory tract is reduced, harmful particles and bacteria entering the lungs will activate macrophages in the bronchial cavity and alveolar cavity and neutrophils and lymphocytes in the lung. While they play the role of phagocytosis, they also release a variety of factors, including the cytokines interleukin- 6 (IL-6), chemokine (KC), and tumor necrosis factor (TNF- $\alpha$ ). These cytokines can destroy the structure of the lung and are important inflammatory factors involved in airway remodeling, leading to the destruction of the lung parenchyma and causing airway stenosis.

In this study, the COPD mice model was established by passive smoking and intratracheal instillation of LPS. Our results showed that there was inflammatory cell infiltration, edema, mucus gland enlargement and mucus secretion, bronchioles expansion and destruction, alveolar cavity enlargement and formation of pulmonary bullae in the lung tissue in the COPD model group. In addition, the number of inflammatory cells in COPD model mice were markedly increased when compared to those in the control group. And the levels of IL-6, KC and TNF- $\alpha$ in the COPD model group were also significantly higher in comparison with those in the control group. Our results were similar to others. Huang Long and others found that TNF- $\alpha$ was higher in the period of COPD than in the stable phase whether smoking or not, and the level of TNF-a was positively correlated with the number of neutrophils and the degree of airflow obstruction in patients with COPD (Huang \& Zheng, 2005). Therefore, they believe that TNF-a is involved in both airway inflammation and airway allergy, which may be related to airway hyper responsiveness, and the degree of airway inflammation may indicate a decrease in the frequency of COPD acute attack. Chen and others reported that the levels of IL-6 in lung were increased in COPD rats (Chen et al., 2016).

JP plaster was a formula combined with many traditional Chinese medicine. Our results showed that the number of inflammatory cells and the levels of IL-6, KC and TNF- $\alpha$ in the COPD model mice were notably decreased after treatment with JP. Furthermore, the results of HE staining also showed that JP significantly improved the pulmonary pathological changes and inflammatory lesions in COPD mice. Those results were similar to the results of others in clinic. It has been reported that Budesonide and formoterol powder inhalation combined with JP plaster significantly improve the lung function of patients with COPD (Huang et al., 2018). Besides, study has showed that JP has therapeutic effect on lung function of children with chronic cough (Chen et al., 2018).

In conclusion, our results showed that JP may have a therapeutic effect on COPD mice through reducing the number of inflammatory cells and the levels of inflammatory factors. JP had a good intervention effect on the progress of COPD to a certain extent. Our research can provide a reliable new method for early clinical treatment and basic research of COPD in stable stage. However, how JP can inhibit the development of COPD by reducing the inflammatory response needs further research.

\section{References}

Chen, S., Li, X., \& Wang, X. (2018). Clinical observation of Jianpi Bufei ointment in the treatment of chronic cough in children with lung and spleen deficiency. Chinese Medicine Guide, 24(15), 75-77.

Chen, X., Shang, L., \& Xie, W. (2016). Effects of Ailuo Kechuanning on IL-6, myeloperoxidase and lung tissue structure in COPD stable period. Modern Distance Education of Chinese Traditional Medicine, 8(16), 141-144.

Dean, E. (2017). Chronic obstructive pulmonary disease. Nursing Older People, 29(4), 12. http://dx.doi.org/10.7748/nop.29.4.12.s14. PMid:28452280.

Hogea, S. P., Tudorache, E., Fildan, A. P., Fira-Mladinescu, O., Marc, M., \& Oancea, C. (2020). Risk factors of chronic obstructive pulmonary disease exacerbations. The Clinical Respiratory Journal, 14(3), 183-197. http://dx.doi.org/10.1111/crj.13129. PMid:31814260.

Huang, L., \& Zheng, Y. (2005). Study on the relationship between airway inflammation and the levels of TNF- $\alpha$ and IL- 8 in sputum in chronic obstructive pulmonary disease. Clinical Medicine of Traditional Chinese Medicine, 12(1), 57.

Huang, Y., Dong, Z., \& Huang, Z. (2018). Clinical observation of Jianpi Bufei ointment combined with western medicine in the treatment of chronic obstructive pulmonary disease in stable stage. Chinese Community Physician, 34(25), 95-96.

Ko, F., \& Sin, D. D. (2020). Twenty-five years of respirology: advances in COPD. Respirology, 25(1), 17-19. http://dx.doi.org/10.1111/ resp.13734. PMid:31840889.

Li, J., Mao, J., Wang, L., Dong, Y., Li, Y., \& Li, S. (2018). Effect of Bufei Jianpi formula on IGF-1, mTOR and HIF1 - $\alpha$ in skeletal muscle of COPD rats. Journal of Traditional Chinese Medicine, 33(02), 268-273.

Li, J., Yang, L., Li, Y., Tian, Y., Li, S., Jiang, S., Wang, Y., \& Li, X. (2015). Metabolomics study on model rats of chronic obstructive pulmonary disease treated with Bu-Fei Jian-Pi. Molecular Medicine Reports, 11(2), 1324-1333. http://dx.doi.org/10.3892/mmr.2014.2843. PMid:25370181.

Li, Y., Li, J. S., Li, W. W., Li, S. Y., Tian, Y. G., Lu, X. F., Jiang, S. L., \& Wang, Y. (2014). Long-term effects of three Tiao-Bu Fei-Shen therapies on NF- $\kappa \mathrm{B} / \mathrm{TGF}-\beta 1 / \mathrm{smad} 2$ signaling in rats with chronic obstructive pulmonary disease. BMC Complementary and Alternative Medicine, 14(1), 140. http://dx.doi.org/10.1186/1472-6882-14-140. PMid:24766819. 
Mao, J., Li, Y., Li, S., Sun, Y., \& Dong, Y. (2018). The effect of Bufei Jianpi formula on the regulation of TNF - $\alpha$ / TNFR / NF - $\kappa$ B pathway on the inflammatory response of skeletal muscle in COPD rats. Chinese Journal of Traditional Chinese Medicine, 33(5), 1863-1867.

Meng, K., Geng, P., \& Zhang, H. (2015). Progress of traditional Chinese medicine treatment in stable phase of chronic obstructive pulmonary disease. Guangming Traditional Chinese Medicine, 21(7), 1583-1585.

Postma, D. S., Bush, A., \& Van den Berge, M. (2015). Risk factors and early origins of chronic obstructive pulmonary disease. Lancet, 385(9971), 899-909. http://dx.doi.org/10.1016/S0140-6736(14)604463. PMid:25123778.

Selzler, A. M., Habash, R., Robson, L., Lenton, E., Goldstein, R., \& Brooks, D. (2020). Self-efficacy and health-related quality of life in chronic obstructive pulmonary disease: a meta-analysis. Patient Education and Counseling, 103(4), 682-692. http://dx.doi.org/10.1016/j. pec.2019.12.003. PMid:31859120.
Song, C., \& Kelsen, S. G. (2013). The relationship between oxidative stress responses and lung inflammation with cigarette smoking. Smoking and Lung Inflammation, 23(7), 99-127. http://dx.doi. org/10.1007/978-1-4614-7351-0_5.

Vidal, A. R., Cansian, R. L., Mello, R. D. O., Kubota, E. H., Demiate, I. M., Zielinski, A. A. F., \& Dornelles, R. C. P. (2020). Effect of ultrasound on the functional and structural properties of hydrolysates of different bovine collagens. Food Science and Technology, 40(2), 346-353. http://dx.doi.org/10.1590/fst.00319.

Wu, J. T., Yang, G. W., Qi, C. H., Zhou, L., Hu, J. G., \& Wang, M. S. (2016). Anti-inflammatory activity of platycodin D on alcohol-induced fatty liver rats via TLR4-MyD88-NF- $\mathrm{BB}$ signal path. African Journal of Traditional, Complementary, and Alternative Medicines, 13(4), 176183. http://dx.doi.org/10.21010/ajtcam.v13i4.23. PMid:28852734.

Zhang, K., Ma, R., \& Li, Z. (2012). Establishment and identification of a chronic obstructive pulmonary disease model in smoking mice. International Journal of Respiration, 32(21), 1607-1611.

Zhong, N. (2011). Chronic obstructive pulmonary disease in China. Chinese Journal of Practical Medicine, 31(5), 321-322. 